\title{
Parlare in pubblico: un'abilità trascurata nella didattica dell'italiano come L1
}

\section{Public speaking: a neglected skill in the teaching of Italian as First language}

\author{
Giuliana Fiorentino \\ Università del Molise \\ $\square$ giuliana.fiorentino@unimol.it
}

Riassunto / L'articolo riflette criticamente sulla didattica del parlato pubblico in Italia auspicando una sua maggiore diffusione. La riflessione si basa da un lato sulla letteratura reperibile in italiano (perlopiù costituita da interventi proposti ai convegni del GISCEL) e dall'altro su un piccolo corpus di parlato pubblico prodotto da studenti universitari in contesto formativo. II quadro che emerge dalla letteratura - che riguarda quasi esclusivamente il contesto scolastico - evidenzia la quasi totale assenza di una didattica e di una riflessione sul parlato in ambito accademico, e rispetto alla didattica del parlato pubblico a scuola sembrerebbe che le proposte siano rimaste in buona misura rare e circoscritte. Il quadro che si può ricavare, pur con molte cautele, dalle prove di parlato pubblico analizzate consente di evidenziare alcuni elementi problematici del parlato degli studenti universitari e di indicare alcuni possibili spunti per un curricolo di didattica del parlato tra scuola e università.

Parole chiave: parlato pubblico; didattica; italiano L1; curricolo.

Abstract / In this article we critically reflect on the didactics of public speaking in Italy, hoping for a wider spread of its practice. These considerations are based on the one hand on the literature available in Italian (mostly made up of interventions proposed at the conferences of GISCEL) and on the other hand on a small corpus of public speaking produced by university students in an educational context. The picture that emerges from the literature - which is related almost exclusively to the school context - high-

\footnotetext{
(C) 2021 Giuliana Fiorentino. Questo è un articolo Open Access, sottoposto a un processo di revisione tra pari a doppio cieco, pubblicato dal Centro competenze didattica dell'italiano lingua di scolarizzazione e dal Servizio risorse didattiche e scientifiche, eventi e comunicazione del DFA-SUPSI in collaborazione con l'Alta scuola pedagogica dei Grigioni. L'articolo è distribuito sotto i termini della Licenza Creative Commons Attribuzione - Non commerciale - Non opere derivate 4.0 Internazionale (CC BY-NC-ND 4.0).
} 
lights the lack of didactics and reflection on speech in the academic context. Moreover, these data highlight the fact that proposals regarding the didactics of public speaking at school seem to be still rare and very limited. The picture that can be cautiously drawn from the public speaking tests analyzed makes it possible to highlight some problematic elements of university students' speaking abilities and to show some possible ideas for a teaching curriculum on speech between school and university.

Keywords: public speaking; teaching; Italian as First language; curriculum.

\section{Introduzione: quale educazione al parlato?}

L'obiettivo di questo lavoro è riflettere sulla esistenza e/o necessità di una didattica esplicita del parlare in pubblico (public speaking) in contesto universitario (e scolastico). La ricerca, che ha un taglio puramente qualitativo basandosi su un piccolo corpus di interazioni orali, intende mostrare alcuni aspetti critici delle performance orali di matricole (quindi tendenzialmente studentesse e studenti appena usciti dal percorso scolastico), per ragionare su metodologie didattiche del parlato e su un possibile curricolo di didattica del parlare in pubblico. I dati su cui si basa questo studio, come si dirà meglio anche più avanti, sono interazioni realizzate da un gruppo di matricole di scienze della comunicazione dell'ateneo in cui insegno (Università del Molise) durante un corso di abilità linguistiche (in parte riconducibili alle cosiddette soft skills) e di introduzione alla linguistica che si svolge subito prima dell'inizio dei corsi universitari curricolari (l'anno accademico di riferimento è il 2020-2021, ma l'esperienza è portata avanti da oltre dieci anni).

Come evidenziato nel titolo, lo scenario in cui ci muoviamo è quello della consapevolezza (ma anche della volontà di verificare in modo più approfondito tale aspetto) di una certa criticità che si riscontra nel parlato di molti studenti universitari, sia che tale abilità riguardi il parlato unidirezionale degli esami orali, sia che riguardi interventi durante le lezioni, sia infine che riguardi rare occasioni di presentazioni orali programmate all'interno delle attività curricolari.

I presupposti di questa analisi sono svariati ed è opportuno richiamarli. Innanzitutto a) l'idea che sia possibile e necessaria una didattica esplicita del parlato; b) inoltre che la didattica esplicita del parlato debba avviarsi fin dalle 
scuole e debba abbracciare diversi tipi di testi orali in modo che la competenza orale degli studenti non si eserciti nelle sole 'interrogazioni' (sull'interrogazione come prova utilizzata con sistematicità nelle scuole italiane si veda Altichieri et al., 1997); c) infine che l'apprendimento delle abilità linguistiche - sia che avvenga seguendo delle regole top-down, sia che si basi sull'esperienza e sull'osservazione critica di materiali altrui e si sviluppi quindi anche per 'copia' e imitazione - vada calato in un addestramento globale alla comunicazione e al potenziamento della competenza comunicativa (per una definizione di questa nozione si veda Hymes, 1972, ma si veda anche Perri, 2018 per un intervento di sintesi critica di tale nozione).

La didattica esplicita del parlato sembra essere abbastanza poco praticata in Italia a livello accademico: le molteplici offerte di corsi di public speaking che è possibile individuare interrogando il web riguardano perlopiù enti di formazione privati o anche solo individui (che si autodefiniscono 'coach'): ad una rapida ricerca sembra che ci sia soltanto un corso offerto da un'università, anch'essa privata, la LUISS, che si inserisce in una introduzione alle cosiddette soft skil$l s^{1}$. Pertanto per discutere di quale possa essere un curricolo di educazione al parlato ci si rivolgerà sostanzialmente a quanto viene proposto nei segmenti scolastici che precedono la formazione universitaria. Del resto questa scelta ha una sua fondatezza se si considera che i dati di parlato su cui si basa questa ricerca sono stati registrati in un corso introduttivo alle abilità linguistiche che viene indirizzato alle matricole, quindi le competenze e abilità degli studenti coinvolti rispecchiano la formazione scolastica ricevuta fino al momento dell'iscrizione all'università.

Spostato quindi il problema dell'educazione al parlato all'analisi dei segmenti di istruzione che precedono la formazione universitaria, per individuare articoli e interventi dedicati a tale tema ci siamo rivolti soprattutto alla produzione editoriale legata ai convegni promossi dal GISCEL, Gruppo di Intervento e Studio nel Campo dell’Educazione Linguistica costituitosi nel 1973, che è «un "intellettuale collettivo" che raccoglie studiosi di linguistica e insegnanti di tutti

1 Il corso insegnato da circa 12 anni presso l'università del Molise non è dedicato esclusivamente al parlato, ma certamente potrebbe annoverarsi tra le poche esperienze di tale didattica a livello accademico. 
gli ordini di scuola interessati agli studi di teoria e descrizione dei fenomeni linguistici e alla pratica educativa nel settore dell'educazione linguistica» (dal sito web dell'associazione https://giscel.it/chi-siamo/).

Nell'ambito dei quaderni del GISCEL ci sono diversi volumi che sono dedicati al parlato, tra questi, segnaliamo (partendo dal più recente e risalendo a ritroso nel tempo) Voghera et al., 2020; De Renzo \& Tempesta, 2014; Piemontese, 2000; Corrà \& Deon, 1997; Brasca \& Zambelli, 1992; Lavinio \& Sobrero, 1991. Ai titoli legati all'attività del GISCEL si possono aggiungere alcuni altri volumi di educazione linguistica i cui curatori e i cui contributi provengono grosso modo dallo stesso gruppo di studiosi che gravitano intorno al GISCEL.

Non abbiamo la pretesa nello spazio di quest'articolo di accennare a tutte le ricerche raccolte in questi volumi: si tratta, in molti casi, soprattutto di descrizioni ragionate di valide esperienze didattiche nel campo dell'educazione al parlato o di descrizioni del parlato degli studenti. Ci limiteremo a presentare i lavori che hanno fornito maggiori stimoli per la presente ricerca.

In un lavoro del 2000 (Brasca \& Ravizza, 2000, p. 113), le autrici individuavano una lista dei tipi di interazione orale in cui sono coinvolti gli studenti nel contesto scolastico. Considerando soltanto quelli in cui sono gli studenti a prendere la parola (quindi escludendo le lezioni e le spiegazioni tenute dai docenti), i tipi si riducono alle interrogazioni, ad altri tipi di esposizioni orali o di relazioni programmate su contenuti disciplinari, alle interazioni col docente e col resto della classe di carattere più libero su argomenti personali o socio culturali o di tipo disciplinare, come ad esempio nel caso di partecipazione interattiva alla spiegazione, alle interazioni tra pari sia di carattere informale (conversazioni su aspetti personali) sia di carattere strutturato come nel caso di lavori di gruppo, discussioni, assemblee. Si fa infine riferimento al parlato e ascolto in situazione di simulazione, e in questo tipo sono menzionate le drammatizzazioni.

Dallo studio citato (Brasca \& Ravizza, 2000) si ricava un'idea centrale, che in parte riguarda anche altre attività scolastiche, e cioè che in buona misura il parlare in pubblico in contesto scolastico sia caratterizzato da scarso 'realismo', scarsa autenticità, e da una significativa riduzione della gamma di finalità che possono caratterizzare la comunicazione orale. L'interazione orale, e la performance dello studente in essa (d'ora in poi ci riferiremo a questo tipo di parlato 
come parlato scolastico) non è infatti valutato nella sua pienezza e quindi anche come prova del livello di competenza raggiunto in quest’abilità, ma serve piuttosto come momento di verifica di saperi e di acquisizione di contenuti. Le correzioni che il docente fa probabilmente riguardano la forma linguistica, e meno spesso si discute della struttura testuale del discorso orale e di come il discorso vada realizzato per raggiungere un certo fine comunicativo. Poiché il fine di molte delle attività di parlato scolastico non è verificare l'acquisizione di questa abilità quanto verificare il possesso di conoscenze disciplinari, le correzioni del docente più probabilmente riguardano la correttezza dei contenuti esposti.

Inoltre se l'interrogazione è condotta dal docente sotto forma di domande che si susseguono secondo uno schema definito dal docente, non è dato modo allo studente di organizzare un discorso e di trovare una propria chiave di presentazione, ma egli si limiterà a rispondere alle richieste e a fare in modo che il contenuto delle risposte rispecchi quanto appreso dai libri o comunque quanto atteso dal docente. Una maggiore autonomia nell'elaborazione e la necessità di produrre un discorso orale persuasivo potrebbero riguardare le presentazioni orali assegnate dai docenti agli studenti, ma il reale spazio e peso che esse hanno nella valutazione dello studente andrebbe chiarita, e comunque anche in questo caso si deve capire se la valutazione riguarda la performance in quanto prova di capacità di parlare in pubblico o in quanto prova di acquisizione e rielaborazione di saperi.

A conferma di un interesse non sempre esplicito per il parlato inteso come abilità, un'abilità che implica diverse competenze e non solo quella linguistica, si veda Lavinio e Sobrero (1991). Il volume, il cui pregio non è qui messo in discussione, s'intitola La lingua degli studenti universitari e presenta una evidente asimmetria nel modo di trattare la scrittura e il parlato degli studenti universitari. È diviso in due parti: la prima parte è dedicata alle forme di scrittura, la seconda a fenomeni del parlato. Mentre nella prima parte (i cui saggi sono molto noti e citati e sono stati anche ampiamente commentati e confrontati da chi scrive con ricerche recenti sulla scrittura degli studenti universitari, cfr. Fiorentino, 2015), affrontando la scrittura, si discute sì di lingua utilizzata negli scritti dagli studenti universitari, ma le questioni linguistiche si legano alle considerazioni sulle tipologie di testi utilizzati nel curricolo accademico (Lavinio, 1991), sui 
corsi per imparare a scrivere all'università (Romanello, 1991), sulle competenze testuali oltre che linguistiche (Tempesta, 1991; Caffi, 1991), nella seconda parte i quattro contributi dedicati al parlato si occupano esclusivamente di lingua e in particolare segnalano in che modo sono realizzati nella lingua parlata degli studenti universitari alcuni fenomeni morfosintattici e lessicali: il lessico tecnico (Berretta, 1991), vari fenomeni di substandard (Bozzone Costa, 1991), le frasi relative (Bernini, 1991), il passivo (Bazzanella, 1991). È interessante osservare che i dati discussi dai quattro autori derivano indifferentemente da materiale registrato all'università durante gli esami, o in occasione di altre interazioni orali, in contesto pur sempre accademico e formale, come discussione di relazioni, tesine e conversazioni con i docenti (Berretta, 1991), oppure da materiale prodotto da studenti universitari in contesto altro, informale (a casa, con amici, ecc.) e quindi si tratta di parlato colloquiale (il caso dei contributi di Bozzone Costa, 1991 e di Bernini, 1991, considerando che quest'ultimo utilizza lo stesso corpus raccolto da Bozzone Costa, 1991); in un ultimo caso (Bazzanella, 1991) il corpus da cui sono estratti i dati è formato da produzioni di studenti in contesto accademico, ma si considerano sia fonti scritte (esami, tesi, ecc.) sia fonti orali (esami, relazioni, ecc.) ${ }^{2}$. Questa asimmetria conferma il quadro che abbiamo tratteggiato per il contesto scolastico, in cui il parlato non viene né 'praticato' come un'abilità a sé da addestrare, esercitare, allenare né di conseguenza studiato come un'abilità che si dispiega in modi diversificati in base al contesto d'uso.

Un paio d'anni più tardi Orletti (1993) proponeva in un breve lavoro alcuni spunti Per una educazione al parlato. Nel suo contributo la studiosa fa cenno alla fioritura di studi sul parlato (a partire dagli anni '80 del secolo scorso) nella linguistica italiana e non soltanto, con la prevalenza di ricerche che, almeno in

2 L'utilizzo di materiale così disomogeneo, che risponde all'interesse di rappresentare, come si evince dal titolo del volume, non tanto la lingua formale ('accademica') usata dagli studenti all'università, ma più semplicemente la lingua degli studenti universitari, va però considerato - in questa sede - un elemento di debolezza di queste analisi o comunque un fattore che, se non attentamente sottolineato, può portare a generalizzazioni infondate e ad altrettanto pericolose sovrapposizioni tra fatti che invece sarebbero meglio riconducibili alla variabilità diafasica, e altri legati alle dimensioni diamesica o anche diastratica. 
Italia, descrivono soprattutto gli aspetti morfosintattici e lessicali grazie ai quali il parlato - utilizzando il codice fonico e appoggiandosi alla multimodalità - si distingue dalla scrittura e dal codice grafico (Orletti, 1993, pp. 65-66). L'attenzione della studiosa però si concentra sulla necessità di costruire «una etnografia dei diversi usi del parlato in una determinata cultura» (Orletti, 1993, p. 66), senza la quale è difficile riconoscere «la rilevanza di un'educazione al parlato come obiettivo dell'educazione linguistica» (p. 66). Il saggio prosegue con una descrizione dei diversi fattori che servono a descrivere un'etnografia dei diversi usi del parlato e che determinano la diversificazione dei tipi di parlato: innanzitutto si distingue tra situazioni a dominanza di parlato e situazioni a dominanza di comportamento non verbale (Orletti, 1993, p. 67); inoltre tra le situazioni a dominanza di parlato si suggerisce di distinguere le diverse situazioni in base al rapporto col destinatario (con la conseguenza di produrre maggiore o minore interattività) e in base alle finalità comunicative (costruzione di relazioni sociali, trasmissione di conoscenze, ad esempio). Le diverse situazioni si correlano alla presenza o assenza di specifici tratti linguistici (lessico, sintassi, e così via). La breve rassegna di possibili situazioni comunicative si chiude con il riferimento al parlato pubblico e alle sue peculiarità, e al fatto che esso è oggetto di un certo mercato di corsi e investimenti in termini di formazione (a livello privato e pubblico soprattutto in ambito aziendale). La studiosa conclude con l'analisi di quali situazioni dovrebbero entrare in un curricolo di educazione al parlato a scuola. Tra queste si suggerisce di affinare l'uso del parlato per trasmettere conoscenze, considerandolo non solo un'abilità linguistica ma un'abilità di studio (Orletti, 1993, p. 72). Prendendo spunto da un volume sull'insegnare a parlare (Brown et al., 1984) Franca Orletti propone alcuni tipi di attività pensate per ragazzi del biennio superiore che possono addestrare a diversi tipi di parlato come la descrizione di relazioni statiche, l'espressione di relazioni dinamiche, l'espressione di relazioni astratte (questi ultimi compiti coprono anche la situazione dei testi argomentativi).

Nonostante qualche elemento di vaghezza che si osserva quando si passa alla parte esemplificativa delle pratiche didattiche e dei suggerimenti, l'articolo di Orletti (1993) è utile per molteplici ragioni, innanzitutto per gli spunti preziosi che offre in merito al tema dell'educazione al parlato, in secondo luogo per 
aver posto la questione in chiave etnografica, un punto di partenza rilevante (e poco chiaro nel mondo scolastico) per identificare la varietà di situazioni e contesti d'uso del parlato. Ma il contributo di Orletti (1993) è utilissimo anche perché ci consente di stabilire che il dibattito sull'educazione al parlato negli anni '90 in Italia era abbastanza fermo, e che non esisteva una pratica educativa in tal senso. Il timore è che poco o nulla sia cambiato dagli anni '90 ad oggi.

Un ultimo spunto per costruire questo percorso di studi italiani che riflettono sull'educazione al parlato è offerto da alcuni saggi contenuti in Piemontese (2000), volume dedicato ai bisogni linguistici delle nuove generazioni. Anche in questo volume gli studi che si occupano del parlato sono minoritari (Milia \& Lecca, 2000; Gruppo di Intervento e Studio nel Campo dell'Educazione Linguistica [GISCEL] Veneto, 2000) a fronte di quelli che analizzano la lingua scritta. Il saggio di Milia e Lecca (2000) si basa a) su interviste semistrutturate fatte a un gruppo di 8 parlanti di età compresa tra i 20 e i 25 anni; b) su un questionario seguito poi da brevi interviste di verifica fatte a un campione di 119 studenti di triennio delle scuole superiori; c) da interviste libere fatte a circa 90 studenti di triennio delle superiori. Da questa indagine le autrici ricavano idee dei giovani intervistati in fatto di lingua, norma, parlato, comunicazione e percezione e autovalutazione delle proprie capacità comunicative. Le autrici rilevano come «occorrerà pure accettare il fatto che giovani come quelli da noi intervistati, che hanno frequentato la scuola media inferiore dopo l'introduzione dei Programmi del '79 che raccomandano ampio spazio al parlato, pare non abbiano avuto grandi occasioni di riflettere sul loro parlato e di migliorarlo senza inseguire la grammatica dello scritto» (Milia \& Lecca, 2000, pp. 52-53). E di nuovo nelle conclusioni osservano come gli studenti non sembrino consapevoli «delle differenze tra modalità orale e scritta della lingua. I criteri con cui analizzano il parlato di compagni/e e insegnanti fanno riferimento soprattutto a categorie del modello grammaticale e dell'analisi logica, e alla forma della lingua scritta» (Milia \& Lecca, 2000, p. 66). L’altro saggio sul parlato contenuto del volume (GISCEL Veneto, 2000) analizza le capacità dimostrate nel parlato nei tre ordini di scuole (primaria, secondaria di primo e secondo grado) per verificare cosa accade lungo il percorso scolastico in particolare in relazione alla qualità linguistica (complessità sintattica, appropriatezza lessicale, ma anche intrica- 
tezza del discorso) dei testi orali prodotti dagli studenti nelle varie fasce di età e alla efficacia comunicativa. Un elemento interessante - ai fini della nostra ricerca - è la panoramica che il saggio del GISCEL Veneto (2000) offre sulle molteplici attività che possono essere proposte a scuola per lavorare e riflettere sul parlato: dal laboratorio proposto nella scuola primaria, alla realizzazione di testi caratterizzati da diverso grado di pianificazione nella scuola superiore di primo grado e di secondo grado. Tra le conclusioni a cui giungono gli autori, è interessante il riferimento al fatto che la mancanza di una chiara percezione da parte degli studenti (ma forse aggiungeremmo noi anche da parte dei docenti) delle specificità dello scritto e del parlato e l'assenza di modelli e di esercitazioni mirate, porta gli studenti a produrre un parlato 'scolastico' poco naturale, e chiaramente modellato sulla norma scritta (GISCEL Veneto, 2000, p. 98). Per questo motivo soprattutto a livello di scuola superiore di primo grado gli autori suggeriscono un addestramento al parlato che spinga gli studenti a produrre testi più chiaramente orientati sul parlato, più autentici, ad esempio caratterizzati da ridondanze, parafrasi, focalizzazioni e riprese (GISCEL Veneto, 2000, p. 100) che rendono più naturale ed efficace un testo orale. Lo spazio individuato per questo addestramento specifico è quello del Laboratorio. Nella scuola superiore si propongono diverse attività, più o meno strutturate, per sviluppare la capacità di parlato pubblico, producendo sia testi referenziali di tipo prettamente scolastico, sia testi argomentativi, sia infine discussioni libere intorno a temi dati. In conclusione l'articolo dimostra un chiaro interesse per la didattica esplicita del parlato, per il suo studio a scuola e per la creazione di una riflessione sui processi che portano alla realizzazione di testi orali efficaci.

Segnaliamo - a conclusione di questa breve rassegna di studi sulla didattica del parlato - il fatto che un discreto interesse per l'abilità dialettica e per l'argomentazione, e quindi per un certo tipo di parlato pubblico, si sta diffondendo più di recente nel mondo scolastico anche italiano, se consideriamo la fioritura di iniziative nelle scuole italiane relativamente al debate che è una metodologia didattica usata in molti paesi europei - anche a livello curricolare - per insegnare agli studenti ad argomentare pro o contro un argomento o un'affermazione misurandosi in squadre in un dibattito ordinato e rispettoso (si veda ad esempio il progetto delle Olimpiadi di debate, presentato nelle pagine del sito 
https://www.debateitalia.it/pagine/il-progetto-1 e l'ulteriore sitografia ivi raccolta https://www.debateitalia.it/pagine/sitografia in cui si trovano riferimenti ad altre iniziative sia nel contesto nazionale sia in quello internazionale).

In sintesi abbiamo cercato di ricostruire il tipo di interesse e attenzione che ha ricevuto l'educazione al parlato nella scuola italiana: il percorso tracciato dalle varie letture citate non pretende di essere esaustivo, ma gli indizi raccolti confermano abbastanza chiaramente il quadro critico a cui abbiamo fatto riferimento a inizio paragrafo. Le ricche e stimolanti esperienze didattiche discusse nei convegni del GISCEL sembrano destinate, dopo 20 anni, a restare ancora rare e legate quasi solo alla buona volontà di singoli docenti motivati (e preparati). I tempi invece sono maturi per diffondere e rendere più pervasive le riflessioni sul parlato che da questi studi abbiamo ricavato, come dimostriamo nei prossimi paragrafi.

\section{II corpus: metodi e risultati}

Il corpus di dati su cui si basa questo studio - come anticipato - è costituito da attività (dialoghi di role-playing) realizzate durante il corso di abilità linguistiche offerto a inizio anno accademico alle matricole di Scienze della Comunicazione dell'università del Molise.

Il corso si svolge in modalità intensiva ( 25 ore in una settimana) ed è un percorso laboratoriale di riflessione sulle situazioni e pratiche comunicative unitamente alle riflessioni sulla lingua e sugli aspetti grammaticali che diventano rilevanti nelle diverse situazioni. Ciascuna delle quattro abilità comunicative (parlare, ascoltare, scrivere, leggere) viene presentata ed esplorata in una giornata di lavoro. Le attività laboratoriali sono proposte come occasione di verifica immediata e opportunità di applicazione delle brevi considerazioni teoriche presentate a inizio giornata e sono ricondotte sempre al contesto accademico. Ad esempio nell'abilità dell'ascolto si propongono videolezioni di livello accademico alle quali poi sono associate l'attività di scrittura di note e appunti o la ricostruzione della scaletta dell'oratore o l'osservazione dell'uso del non verbale da parte di oratori esperti. Le videolezioni di argomento disciplinare vengono poi osservate dal punto di vista linguistico, ad esempio con considerazioni sul linguaggio specialistico (con riferimento al lessico), sui connettivi, sull'uso di 
marche discorsive che organizzano e tengono coeso il discorso orale. I contenuti sono scelti tra quelli vicini al particolare corso di studi: lo sforzo è di creare un contesto di lavoro motivante e realistico, indicando l'utilità e la spendibilità immediata di quanto appreso nel contesto di studio che le matricole stanno per intraprendere.

Per quanto riguarda il parlato le attività che vengono proposte sono molteplici e gli scopi da raggiungere altrettanto ampi. L'obiettivo fondamentale - dato il tempo limitato - è di sollecitare la curiosità e riflessione sul parlato in quanto abilità autonoma e dotata di sue peculiarità rispetto allo scritto. La modalità con cui vengono proposte le attività è progressiva (da attività che richiedono minore preparazione ad altre via via più pianificate) e il clima è in parte ludico o comunque si mira a suscitare interesse e a presentare le attività come sfide linguistico-cognitive da risolvere.

Le attività di parlato iniziano col richiedere un breve discorso di autopresentazione alla classe, imponendo un numero limitato di secondi di durata (massimo 60). Gli studenti hanno qualche minuto per prepararsi, ma spesso si accorgono di non riuscire neppure a 'occupare' l'intero spazio assegnato, pur trattandosi di un compito piuttosto facile (parlare di sé). Successivamente lo stesso compito viene svolto con un tema assegnato (motivare la scelta del percorso di studi, oppure la scelta dell'ateneo molisano, pro e contro, ecc.) e viene data la possibilità di scrivere una scaletta. Successivamente ancora si propone di svolgere la stessa attività non davanti al gruppo di colleghi del corso, ma registrando in solitudine un file audio che verrà poi ascoltato dalla classe. Infine si propongono attività di esposizione orale di contenuti disciplinari letti oppure ascoltati. Accanto al parlato monologico si sollecitano attività dialogiche del genere utilizzato anche nella didattica delle lingue straniere (il role playing) per valutare soprattutto l'efficacia comunicativa e la capacità di riuscire convincenti e spontanei.

Intorno a ciascuna delle attività svolte il docente sollecita la riflessione degli studenti e li spinge ad analizzare le performance ascoltate sulla base di alcuni parametri preventivamente condivisi con loro. Si invitano i partecipanti a confrontare le diverse performance osservando in che modo influiscono i diversi elementi di contesto: ad esempio si confronta il parlato a braccio con il par- 
lato basato su una scaletta; il tema libero o comunque personale con il tema preassegnato e su contenuti di studio; l'esposizione davanti al pubblico con la registrazione in solitudine di un file audio. Gli studenti sono guidati a rendersi conto degli aspetti linguistici come la correttezza di un discorso oppure la selezione lessicale. Si osserva in che modo chi parla gestisce la comunicazione non verbale (posture, tic, dondolii, mimica facciale, ecc.) o quella paraverbale (intonazione e ritmo dell'eloquio), inoltre si valutano l'originalità dei contenuti e la felicità pragmatico-comunicativa dei discorsi prodotti.

L’analisi del parlato su cui ci concentriamo è quella dei dialoghi di roleplaying. I criteri di analisi sono i cinque che abbiamo elencato sopra: a) analisi linguistica (correttezza, scelte lessicali); b) gestione del non verbale (posture, tic, dondolii, mimica facciale, ecc.); c) gestione del paraverbale (intonazione e ritmo dell'eloquio); d) originalità tematica; e) felicità pragmatico-comunicativa del testo prodotto.

Durante il corso anche gli studenti che ascoltano sono invitati a commentare e discutere le performance dei colleghi in modo che l'attenzione sia mantenuta alta e allo stesso tempo ci si abitui a un dialogo tra pari sugli apprendimenti. La consegna del compito è la seguente:

\section{$\mathrm{T} 1$}

Role playing: attività che si svolge a coppie. I due studenti realizzano un colloquio di lavoro di circa 2 minuti: il datore di lavoro rivolge le domande all'aspirante lavoratore. Le posizioni lavorative sono preassegnate a partire da un elenco dato (barista, babysitter, dogsitter, buttafuori, receptionist, cameriere, animatore, bagnino, commesso/a ecc.) e sono legate perlopiù al genere di lavori che spesso svolgono proprio studentesse e studenti. L'obiettivo principale è che l'aspirante lavoratore/ lavoratrice convinca il datore / la datrice di lavoro di essere il/la migliore candidato/a per quel lavoro.

I dialoghi che abbiamo trascritto sono in tutto dieci e si sono svolti in un'ora di lezione. La trascrizione corrisponde a un corpus di 2.600 parole circa. Sottoponendo il corpus nella sua interezza all'analisi automatica (abbiamo utilizzato sia il sito www.corrige.it che assegna un indice di leggibilità basato sull'applicazione della formula di Gulpease sia il demo di Read.it, consultabile alla pagina http://www.italianlp.it/demo/read-it/, che è uno «strumento di valutazio- 
ne della leggibilità per quanto riguarda l'italiano, che combina le tradizionali caratteristiche del testo grezzo con informazioni lessicali, morfo-sintattiche e sintattiche») abbiamo ricavato alcune informazioni di carattere generale sulla lingua dei dialoghi.

Il testo ha un indice medio di leggibilità pari a 56/100 che significa un testo facile per chi ha un diploma superiore, difficile per chi ha la licenza media, molto difficile per chi ha la licenza elementare. Ė costituito da 121 periodi, con una media di 21,6 parole per periodo. Le parole sono mediamente di cinque caratteri. I periodi sono articolati mediamente in 3,5 proposizioni, con un'alta prevalenza di principali $(90,2 \%)$ sulle subordinate (9,8\%); le proposizioni sono mediamente fatte di 6,2 parole. Dal punto di vista lessicale il testo presenta un 78\% di lemmi del Vocabolario di Base, VdB (secondo l'analisi di Corrige il 93\% dei lemmi appartiene al VdB) con una type/tokens ratio abbastanza alta, pari al $60 \%{ }^{3}$.

Oltre ai pochi dati estratti automaticamente possiamo aggiungere che i testi risultano abbastanza fluidi linguisticamente, ma caratterizzati dalle tipiche disfluenze del parlato come allungamenti di vocale, qualche riempitivo come allora a inizio turno, soprattutto a inizio dialogo, ma in quantità neppure eccessiva, e da qualche caso di falsa partenza o di riformulazione; esempi in (1) e (2):

(1) [B] no credo sia un lavoro molto an- / nonostante sia molto impegnativo anche molto divertente ${ }^{4}$

(2) $[\mathrm{A}]$ allora salve ho letto un annuncio riguardo delle lezioni private e vorrei sapere in che materia / di quali materie si occupa / cioè quali materie offre

3 Il dato non è ritenuto del tutto affidabile se si considera che, come evidenziato da vari studi (cfr. Heaps, 1978; Tweedie \& Baayen, 1998; Covington \& McFall, 2010), la percentuale di parole uniche rilevate in un corpus è molto influenzata dalle dimensioni del corpus stesso (ovvero dal numero di tokens): corpora più piccoli hanno una TTR più alta. Il calcolo della TTR permette comunque di ottenere indicazioni di massima sul grado di ricchezza lessicale del testo analizzato.

4 I dialoghi sono trascritti in ortografia italiana con pochissimi diacritici per renderli altamente leggibili. Si usa talvolta la sbarra obliqua per segnalare una nuova unità sintattica. A è stato usato per indicare i turni del datore di lavoro e B per quelli dei candidati. 
Mentre i primi studenti mostrano un certo imbarazzo soprattutto nell'avvio del dialogo, si veda l'esempio in cui ciò è più evidente:

(3) [A] eh oddio non so come iniziare eh buonasera lei ha chiamato per un colloquio?

Nell'arco dell'ora, forse anche avvalendosi delle osservazioni critiche che vengono via via mosse a chi ha già presentato il dialogo, gli studenti successivi appaiono più spigliati e questo si osserva anche in alcune scelte come una migliore organizzazione di aspetti testuali, ad esempio l'aperura e chiusura delle conversazioni con saluti iniziali (4) e finali (5) e (6) abbastanza tipici del genere testuale intervista conoscitiva in contesto lavorativo:

(4) [A] buonasera

[B] buonasera

(5) $[\mathrm{A}]$ va bene le farò sapere al più presto

[B] va bene grazie

(6) $[\mathrm{A}]$ va benissimo le faremo sapere

[B] La ringrazio buona giornata

Perlopiù gli studenti che svolgono il ruolo di datori di lavoro si rivolgono con il Lei all'interlocutore, ma in un dialogo si passa dal Lei al Tu, per poi ritornare al Lei.

Per quanto concerne l'analisi linguistica possiamo aggiungere che alcuni errori rilevati nei dialoghi riguardano le scelte lessicali, come nel caso riportato in (7) dove la studentessa utilizza il termine stazza invece di taglia, riferendosi a un cane:

(7) $[\mathrm{B}]$ e ho lavorato sia con cani di grande stazza sia con cani anche più piccoli quindi credo di poter lavorare con lei

In qualche caso la scelta lessicale può essere contestata per un eccesso di informalità, come nell'esempio riportato in (8) dove viene utilizzato il verbo stare dietro a: 
(8) [A] allora lei mi ha detto che ha già avuto esperienza ha lavorato in altri asili sa che cosa significhi stare dietro a dei bambini

Nello stesso dialogo, peraltro, la stessa studentessa usa un'espressione molto più adeguata:

(9) [A] no un asilo per l'infanzia noi cerchiamo qualcuno che accudisca i bambini in un asilo

L'uso di espressioni poco formali (alcune altre evidenziate in corsivo negli estratti successivi, (ad esempio nell'estratto riportato come (16)) anche la scelta del salve come formula di saluto sono elementi che si collocano nella stessa tipologia di 'errore', cioè l'adozione di un registro troppo informale e che rischia in un attimo di risultare substandard (come il caso di frequentare ripetizioni dell'estratto (16)).

In un altro caso la scelta lessicale inappropriata nasconde forse la scarsa conoscenza di ciò di cui si parla, come nel caso riportato in (10) dove non si può parlare genericamente di arti marziali, dato che le discipline sono molte e ciascuna misura a proprio modo il livello di perizia raggiunto da chi si allena e non necessariamente la cintura nera costituisce il livello massimo:

(10) [B] sono una delle persone più qualificate in questo campo perché sono vincitrice per tre volte del primo premio regionale per quanto riguarda il body building e sono cintura nera in arti marziali

Per quanto riguarda la gestione del non verbale (posture, tic, dondolii, mimica facciale, ecc.) poiché il corso si è svolto online non è stato possibile osservare tutti i parametri: in particolare non si sono potute valutare pienamente la postura dei due interlocutori - che si vedevano solo online in piattaforma - e la gesticolazione. In qualche caso è stato fatta notare l'adozione di movimenti inappropriati (dondolio del busto oppure il toccarsi continuamente il viso).

Un aspetto interessante rilevato attraverso l'osservazione della mimica facciale è che questo è il canale che meglio tradisce - con sorrisi e sguardi divertiti 
- la consapevolezza da parte degli studenti che si stanno simulando situazioni e contenuti non reali, laddove invece il canale verbale cerca di calarsi - con maggiore o minore efficacia - nei ruoli assegnati.

Per quanto concerne la gestione del paraverbale (intonazione e ritmo dell'eloquio) si osserva che talvolta il dialogo risulta molto lento, tradendo il lavoro di pianificazione non del tutto fluido, e non del tutto naturale. Questo secondo aspetto di nuovo ha a che fare con la consapevolezza di essere in contesto di simulazione.

Se però è accettabile che persone non abituate a recitare possano provare imbarazzo e dimostrare scarsa perizia nel simulare situazioni e ruoli non reali, magari mai vissuti (soprattutto nel ruolo di datore di lavoro), meno accettabile è il fatto che nei dialoghi si evidenzino aspetti contenutistici e pragmatici di scarsa efficacia e appropriatezza. In questo caso infatti gli studenti avendo avuto un certo preavviso e avendo ricevuto istruzioni dettagliate avrebbero potuto pianificare un dialogo verosimile.

Dal momento che il corpus è limitato, presentiamo - in un modo che non può che essere poco sistematico - alcune delle questioni più interessanti che sono emerse.

Ad esempio dal punto di vista della struttura del dialogo si osserva che in quasi tutti è omessa la richiesta al candidato di presentarsi facendo riferimento a propri titoli, curriculum, esperienza. Unica eccezione la seguente, dove però la richiesta è formulata in modo generico:

(11) [A] buonasera lei è qui per il colloquio

[B] sì salve buonasera Sono la signora XXXX piacere

[A] piacere mi parli di lei

Questo dà modo all'interlocutore di divagare in modo spesso poco pertinente, ed infatti la risposta a (11), che riportiamo come estratto (12), è poco centrata sulla presentazione delle proprie qualità:

(12) $[B]$ allora io ho visto il vostro annuncio come animatore per animatrice in questo caso per il vostro villaggio sarà la mia prima esperienza perché ho avuto 
altri tipi di lavoro però siccome mi sono sempre rimessa in gioco e mi piace anche comunque il contatto con le persone ho deciso di propormi per insomma per questo lavoro che voi richiedete che lei richiede

L'assenza di uno schema chiaro ricavabile dai dialoghi realizzati (le interviste iniziano in modo diverso e spesso le domande sembrano estemporanee, si veda ad esempio l'attacco dell'estratto (13)) è forse l'elemento di testualità che meglio dimostra la scarsa abitudine a pianificare i discorsi. Non si è avuto modo di discutere esplicitamente di questo aspetto con gli studenti, ma la sensazione è che ad alcuni almeno possa sfuggire del tutto che anche un discorso si pianifica sulla base di un piano testuale tipico del genere testuale che si sta realizzando. La studentessa che apre il dialogo con il turno riportato nell'estratto (14) ad esempio è l'unica a inserire nell'esordio i requisiti imprescindibili previsti dal datore di lavoro:

(13) [A] allora ho ricevuto la sua candidatura per il lavoro di buttafuori e ne ho preso visione volevo per prima cosa chiederle perché ha scelto questo lavoro che è prettamente maschile e se si ritrova in quelli che sono i requisiti fisici per affrontare questo lavoro

(14) [A] Io mi chiamo Giovanna e sono la direttrice di quest'hotel abbiamo ricevuto tramite mail il suo curriculum e le vorrei far sapere che ci sono tre requisiti fondamentali ovvero avere un diploma avere almeno 18 anni e saper parlare bene inglese e almeno un'altra lingua mi parli brevemente di lei

Un aspetto molto carente è quello delle motivazioni: in almeno due casi la motivazione a candidarsi per una certa posizione lavorativa è legata paradossalmente all'inesperienza e quindi alla voglia di fare nuove esperienze, al rimettersi in gioco (come nell'estratto (12) già visto), o addirittura al desiderio di migliorare il proprio ‘carattere' (15):

(15) [A] Come mai è interessata a questo lavoro

[B] perché sono una persona introversa e credo che il lavoro di animatrice possa aiutarmi a migliorare questo aspetto del mio carattere [A] capisco e ha esperienze passate cioè ha avuto esperienze già in questo campo 
[B] no questa sarebbe la prima volta

[A] ho capito quindi il tuo unico motivo è diciamo migliorare questo tuo aspetto caratteriale oppure hai anche altre motivazioni

Allo stesso modo risulta complicato individuare e riferire i propri punti di forza, senza cadere nella ripetizione dei concetti o anche in una pericolosa banalità:

(16) $[\mathrm{B}]$ secondo me il vantaggio che posso offrire a un eventuale alunno è che anche io ai tempi delle superiori ho frequentato delle ripetizioni (sic!) e quindi so come un alunno vuole che ci si interfacci con lui e anche magari come non rendere troppo pesante il metodo di studio quindi semplificando l'apprendimento diciamo

[A] che cosa credi di avere rispetto agli altri ragazzi che offrono ripetizioni / hai un buon livello di preparazione?

[B] sì però appunto più che altro penso di avere una certa empatia con gli studenti che hanno bisogno di ripetizioni proprio perché anche io ai tempi ero sulla stessa situazione (sic!)

(17) [A] mi elenchi il suo principale punto di forza il suo principale punto di debolezza

[B] il mio punto di forza sicuramente - che in questo caso potrebbe essere fondamentale - è la spiccata capacità di lavorare in team, nel team / punto di debolezza per per ora a parte l'orario perché sono comunque mamma non penso di avere punti deboli

Alle volte infine ci sono delle lampanti incongruenze, come la domanda che si trova in almeno tre dialoghi - di quali siano le preferenze di orario dell'aspirante lavoratore (normalmente è il datore di lavoro che stabilisce l'orario in base alle sue esigenze):

(18) [A] Ok è disponibile in tutte le fasce orarie o ha delle preferenze

(19) [A] bene e riguardo agli orari lei ha delle preferenze perché questo lavoro almeno nel mio locale è prettamente notturno quindi vorrei sapere se lei ha delle difficoltà a lavorare in orari notturni 
Pur nella consapevolezza di aver tracciato solo qualche spunto di riflessione per ricerche che dovrebbero essere condotte in modo certamente più sistematico e con un corpus di ampio respiro, meglio strutturato e un campione di studentesse e studenti più rappresentativo, crediamo che il lavoro proposto possa avere una sua utilità. Le considerazioni introduttive (paragrafo 1) e le osservazioni sui dati linguistici (paragrafo 2) sono tristemente coerenti: ad una mancanza o assenza totale di didattica esplicita relativamente all'abilità del parlare e di riflessione sulle specificità linguistiche del parlato, che caratterizza in buona misura l'esperienza scolastica delle nostre matricole, corrisponde una certa incapacità a gestire appropriatamente nell'oralità testi relativamente semplici e compiti comunicativi abbastanza circoscritti.

L'esperienza maturata nel tempo durante il corso di abilità linguistiche dimostra però che è possibile osservare dei miglioramenti, anche piccoli - già considerando le attività iniziali e confrontandole con quelle successive. I miglioramenti si possono valutare nella qualità dei discorsi prodotti e rispecchiano un aumento di consapevolezza da parte degli studenti, frutto dell'impegno didattico che curi esplicitamente il parlato.

\section{Bibliografia}

Altichieri, L., Bonetti, L., Iannacci, P., \& Ridolfi G. (1997). «Parlar bene» a scuola: dal parlare al parlato formale nella scuola media. In L. Corrà \& V. Deon (a cura di), "Maxima debetur puero reverentia". Esperienze di interazione verbale in classe (pp. 89-127). Firenze: La Nuova Italia.

Bazzanella, C. (1991). Il passivo nella produzione scritta e orale: forme e funzioni. In C. Lavinio \& A. A. Sobrero (a cura di), La lingua degli studenti universitari (pp. 189-212). Firenze: La Nuova Italia.

Bernini, G. (1991). Frasi relative nel parlato colloquiale. In C. Lavinio \& A. A. Sobrero (a cura di), La lingua degli studenti universitari (pp. 165-187). Firenze: La Nuova Italia.

Bozzone Costa, R. (1991). Tratti substandard nel parlato colloquiale. In C. Lavinio \& A. A. Sobrero (a cura di), La lingua degli studenti universitari (pp. 123163). Firenze: La Nuova Italia. 
Brasca, L., \& Ravizza, G. (2000). Didattica dell'italiano: le abilità linguistiche. In D. Bertocchi, L. Brasca, S. Citterio, D. Corno \& G. Ravizza (a cura di), Insegnare italiano. Un curricolo di educazione linguistica (pp. 88-173). Firenze: La Nuova Italia.

Brasca, L., \& Zambelli, M. L. (a cura di) (1992). Grammatica del parlare e dell'ascoltare a scuola. Firenze: La Nuova Italia.

Brown, G., Anderson A., Shillcock R., \& Yule G. (1984). Teaching Talk. Cambridge: CUP.

Caffi, C. (1991). Aspetti pragmatici e testuali delle introduzioni a tesi di laurea e specializzazione in materie scientifiche. In C. Lavinio \& A. A. Sobrero (a cura di), La lingua degli studenti universitari (pp. 71-98). Firenze: La Nuova Italia.

Corrà, L., \& Deon, V. (a cura di) (1997). "Maxima debetur puero reverentia". Esperienze di interazione verbale in classe. Firenze: La Nuova Italia.

Covington, M. A., \& McFall, J. D. (2010). Cutting the Gordian knot: The moving-average type-token ratio (MATTR). Journal of Quantitative Linguistics. 17, 94-100.

De Renzo, F., \& Tempesta, I. (a cura di) (2014). Il parlato a scuola - Indicazioni per il primo ciclo d'istruzione. Roma: Aracne.

Fiorentino, G. (2015). Aspetti problematici del discorso accademico: un'analisi dei riassunti delle tesi di laurea. Cuadernos de Filología Italiana. 22, 263284. http://dx.doi.org/10.5209/rev CFIT.2015.v22.50961

GISCEL Veneto (2000). Competenza sintattica in testi orali di tipo formale e autopercezione degli studenti nella scuola elementare, media e superiore. In E. Piemontese (a cura di), I bisogni linguistici delle nuove generazioni (pp. 91116). Firenze: La Nuova Italia.

Heaps, H. S. (1978). Information retrieval: Computational and theoretical aspects. Orlando, FL: Academic Press. 
Hymes, D. (1972). On communicative competence. In J. B. Pride \& J. Holmes (a cura di), Sociolinguistics (pp. 269-293). Harmondsworth: Penguin.

Lavinio, C. (1991). I problemi della scrittura. In C. Lavinio \& A. A. Sobrero (a cura di), La lingua degli studenti universitari (pp. 13-30). Firenze: La Nuova Italia.

Lavinio, C., \& Sobrero, A. A. (a cura di) (1991). La lingua degli studenti universitari. Firenze: La Nuova Italia.

Milia, L., \& Lecca, M. T. (2000). Problemi di autopercezione e di valutazione della comunicazione spontanea dei giovani. In E. Piemontese (a cura di), I bisogni linguistici delle nuove generazioni (pp. 39-69). Firenze: La Nuova Italia.

Orletti, F. (1993). Per una educazione al parlato. In F. Orletti (a cura di), L'educazione linguistica nella scuola superiore: un itinerario formativo (pp. 63-77). Firenze: La Nuova Italia.

Perri, A. (2018). La competenza comunicativa: mezzo secolo di una nozione scomoda. Rivista di psicolinguistica applicata. XVIII, 97-112.

Piemontese, E. (a cura di) (2000). I bisogni linguistici delle nuove generazioni. Firenze: La Nuova Italia.

Romanello, M. T. (1991). Imparare a scrivere all'università. In C. Lavinio \& A. A. Sobrero (a cura di), La lingua degli studenti universitari (pp. 31-45). Firenze: La Nuova Italia.

Sobrero, A. A. (1991). Prefazione. In C. Lavinio \& A. A. Sobrero (a cura di), La lingua degli studenti universitari (pp. 1-9). Firenze: La Nuova Italia.

Tweedie, F. J., \& Baayen, R. H. (1998). How variable may a constant be? Measures of lexical richness in perspective. Computers and the Humanities. 32, 323-352.

Voghera, M., Maturi, P., \& Rosi, F. (a cura di) (2020). Orale e scritto, verbale e non verbale: la multimodalità nell'ora di lezione. Firenze: Cesati. 